\title{
Enhanced peripheral visual processing in congenitally deaf humans is supported by multiple brain regions, including primary auditory cortex
}

\author{
Gregory D. Scott ${ }^{1,2+}$, Christina M. Karns ${ }^{1+}$, Mark W. Dow ${ }^{1}$, Courtney Stevens ${ }^{3}$ and Helen J. Neville ${ }^{1 *}$ \\ ${ }_{1}^{1}$ Brain Development Lab, Department of Psychology, University of Oregon, Eugene, OR, USA \\ ${ }^{2}$ Division of Pulmonary and Critical Care Medicine, Department of Medicine, Oregon Health and Science University, Portland, OR, USA \\ ${ }^{3}$ Department of Psychology, Willamette University, Salem, OR, USA
}

\author{
Edited by: \\ François Champoux, Université de \\ Montréal, Canada \\ Reviewed by: \\ Francesco Pavani, University of \\ Trento, Italy \\ Matthew William Geoffrey Dye, \\ University of Illinois at \\ Urbana-Champaign, USA \\ *Correspondence: \\ Helen J. Neville, Brain Development \\ Lab, Department of Psychology, \\ University of Oregon, Eugene OR \\ 97403-1227, USA \\ e-mail:neville@uoregon.edu \\ ${ }^{\dagger}$ These authors have contributed \\ equally to this work.
}

Brain reorganization associated with altered sensory experience clarifies the critical role of neuroplasticity in development. An example is enhanced peripheral visual processing associated with congenital deafness, but the neural systems supporting this have not been fully characterized. A gap in our understanding of deafness-enhanced peripheral vision is the contribution of primary auditory cortex. Previous studies of auditory cortex that use anatomical normalization across participants were limited by inter-subject variability of Heschl's gyrus. In addition to reorganized auditory cortex (cross-modal plasticity), a second gap in our understanding is the contribution of altered modality-specific cortices (visual intramodal plasticity in this case), as well as supramodal and multisensory cortices, especially when target detection is required across contrasts. Here we address these gaps by comparing $\mathrm{fMRI}$ signal change for peripheral vs. perifoveal visual stimulation $\left(11-15^{\circ}\right.$ vs. $\left.2-7^{\circ}\right)$ in congenitally deaf and hearing participants in a blocked experimental design with two analytical approaches: a Heschl's gyrus region of interest analysis and a whole brain analysis. Our results using individually-defined primary auditory cortex (Heschl's gyrus) indicate that fMRI signal change for more peripheral stimuli was greater than perifoveal in deaf but not in hearing participants. Whole-brain analyses revealed differences between deaf and hearing participants for peripheral vs. perifoveal visual processing in extrastriate visual cortex including primary auditory cortex, $M T+N 5$, superior-temporal auditory, and multisensory and/or supramodal regions, such as posterior parietal cortex (PPC), frontal eye fields, anterior cingulate, and supplementary eye fields. Overall, these data demonstrate the contribution of neuroplasticity in multiple systems including primary auditory cortex, supramodal, and multisensory regions, to altered visual processing in congenitally deaf adults.

Keywords: visual attention, deaf, human, fMRI, Heschl's gyrus, auditory cortex

\section{INTRODUCTION}

A number of studies report alterations in visual performance following deafness (Bavelier and Neville, 2002; reviewed in Bavelier et al., 2006). In general, deaf individuals show enhanced motion detection, visual orienting, and selective attention in peripheral but not central visual fields (Neville and Lawson, 1987b; Loke and Song, 1991; Bosworth and Dobkins, 2002a; Stevens and Neville, 2006) (but see Bottari et al., 2011). Several studies have examined the neural substrates supporting these enhancements. In deaf cats, visual enhancements rely on specific regions of the auditory cortex serving a homologous function (Lomber et al., 2010). Data from adult humans suggest that both intramodal plasticity and supramodal cortex also contribute to enhanced peripheral visual processing. For example, neuroimaging studies of visual processing in congenitally deaf humans show increased responses in visual cortex (e.g., motion sensitive MT+/V5) and attention-related brain networks in tasks using peripheral visual stimuli (Bavelier et al., 2001; Bavelier and
Neville, 2002). Previous studies suggest this these alterations may be partially accounted for by increased attention to the periphery in deaf participants (Neville et al., 1983; Neville and Lawson, 1987a,b; Bavelier et al., 2000, 2001).

CROSS-MODAL NEUROPLASTICITY OF PRIMARY AUDITORY CORTEX

A key question in cross-modal plasticity is whether congenital deafness affects the organization of low-level primary sensory cortex or whether these specialized brain regions are exempt from neuroplasticity. Many studies testing this question have reported no or non-specific responses to visual stimuli in primary auditory cortex (Vachon et al., 2013; see Bavelier et al., 2006 for a review). In contrast, a few studies have suggested cross-modal plasticity of primary auditory cortex (visual motion stimuli: Finney et al., 2001; vibrotactile stimuli: Auer et al., 2007). The main limitation of these studies is that the site of human primary auditory cortex, Heschl's gyrus, is highly variable in its morphology across individuals and approaches that use spatial averaging 
across participants and identification based on atlases suffer from poor localization and potential loss of power due to low spatial overlap across participants. We recently addressed this using individual anatomically-defined Heschl's gyrus regions of interest and showed that Heschl's gyrus responds to both touch and vision in congenitally deaf adults, and further, the cross-modal responses in primary auditory cortex were larger contralateral to stimulation (Karns et al., 2012).

It is important to begin comparing findings in humans with findings in animal models that allow for regional specificity in terms of cytoarchitecture and function. For example some animal studies indicate that primary auditory cortex or closely related regions respond to vision and somatosensation in early deafness (Allman et al., 2009; Meredith et al., 2009; Lomber et al., 2010; Meredith and Lomber, 2011), while other studies report deaf primary auditory cortex does not respond to vision (Kral et al., 2003). Comparisons across human and animal models are complicated by imprecise delineation of low-level auditory cortex in humans (Hackett, 2011) but progress is being made. For example, according to a recent tonotopic fMRI study the anterior aspect of Heschl's likely corresponds mainly to human A1, while the posterior aspect corresponds to human area $\mathrm{R}$ (Da Costa et al., 2011). Both $\mathrm{A} 1$ and $\mathrm{R}$ are core auditory regions in the macaque monkey (Hackett, 2011). Although tonotopy is not possible in deaf populations, in the current study we parcellate Heschl's gyrus into its anatomical anterior and posterior divisions (putative analog to primate areas $\mathrm{A} 1$ and $\mathrm{R}$, respectively) to contribute to cross-species comparisons and to test the hypothesis that enhanced peripheral vision in deafness is reflected by enhanced visual responses of peripheral vs. perifoveal visual stimulation in deaf primary auditory cortex.

\section{ADAPTATION AND PLASTICITY IN OTHER BRAIN REGIONS}

Although a region of interest approach is important for assessing plasticity in brain areas with high anatomical variability like Heschl's gyrus, a strength of human fMRI is the ability to obtain whole brain coverage with reasonable spatial specificity. Using these whole-brain approaches, neuroimaging studies of motionand attention-related visual processing in deaf humans have revealed increased responses in brain regions involved as both the sources of attention modulation (i.e., FEF, PPC, post-STS) as well as likely sites of attentional modulation (MT+, V1/V2, V3a), including higher-level extrastriate visual cortex (e.g., MT+/V5) (Bavelier et al., 2000, 2001). However, it is difficult to determine whether these enhancements occur with simple visual stimuli presented in the periphery or whether such plasticity requires higher-level stimulus properties (e.g., shape, motion) or high attention load. To address these questions, we performed whole brain group analyses in deaf and hearing participants comparing brain responses to simple point-light stimuli presented in the peripheral vs. perifoveal visual field.

In summary, the primary goal of the current study was to determine whether primary auditory cortex supports enhanced visual processing of the visual periphery in profoundly, congenitally deaf adults. In addition, we sought to determine whether multisensory and/or supramodal cortices support enhanced peripheral visual processing in congenitally deaf humans when simple visual stimuli are presented in a low-level visual detection task. To do this, in the present study we combined region-ofinterest, individual parcellation techniques to measure responses within primary auditory cortex (Heschl's gyrus), along with whole brain group analysis comparing deaf and hearing participants to perifoveal vs. peripheral visual stimulation using a target detection task.

\section{MATERIALS AND METHODS PARTICIPANTS}

Ten congenitally and profoundly deaf adults (six females) and seven hearing adults (five females) participated in the current study. Participant ages ranged from 19- to 45-years-old (Deaf: mean age 30 years \pm 7.6 , range $19-45$, Hearing: mean age 30 years \pm 10.6 , range $20-45)$. One deaf participant was excluded from final analysis due to motion-related artifacts. All deaf participants reported being profoundly and congenitally deaf due to heredity. Audiogram data, available from four of the nine deaf participants, confirmed profound deafness, with a mean hearing level of $100 \mathrm{~dB}$ (range 91-110 dB). All deaf participants were also native users of American Sign Language. All research was performed with the written informed consent of participants and in compliance with the Human Subjects Institutional Review Board at the University of Oregon.

\section{APPARATUS}

An MRI video projection apparatus was used to present single, yellow discrete disk stimuli on a video screen at eccentricities of $2,4,7,9,11,13$, and $15^{\circ}$ (Figure 1). Stimuli were presented via an InFocus LP350 video system viewed by the subject through a mirror mounted on the MRI head coil. A dim red light was continuously present at fixation (Figure 1). All stimuli were presented along polar angle radials $45^{\circ}$ above and below the horizontal meridian in the right visual field. We focused on the right visual field due to fMRI time constraints; the left eye was patched to reduce visual fatigue. Similar discrete stimulus presentation has been used in previous retinotopic experiments (Tootell et al., 1998a,b; Di Russo et al., 2001; Bridge et al., 2005; Lu et al., 2005). Stimuli flashed at $8 \mathrm{~Hz}$ (standards: $85 \%$ probable) or $14 \mathrm{~Hz}$ (targets: 15\% probable) for $3.2 \mathrm{~s}$ before changing location in a pseudo-random sequence optimizing the visual distance of sequential stimuli. Stimulus timing was controlled by Presentation software (Neurobehavioral Systems).

\section{TASK}

The participants' task was to detect visual stimuli that flickered at a faster rate $(14 \mathrm{~Hz})$ than standard stimuli $(8 \mathrm{~Hz})$. Participants pressed a keypad in response to the target stimuli while maintaining central fixation and attending to the entire right visual field. Prior to scanning, participants practiced the task using a computer simulation outside the magnet room until they were able to accurately detect every target. During the scan, researchers monitored subject compliance to response and fixation instructions using on-line response checking and an ASL 5000/LRO infrared eye tracker (Applied Science Laboratories), although behavioral responses were not able to be recorded due to an error in the experimental apparatus. 
MRI video projection from $2^{\circ}$ to $14^{\circ}$

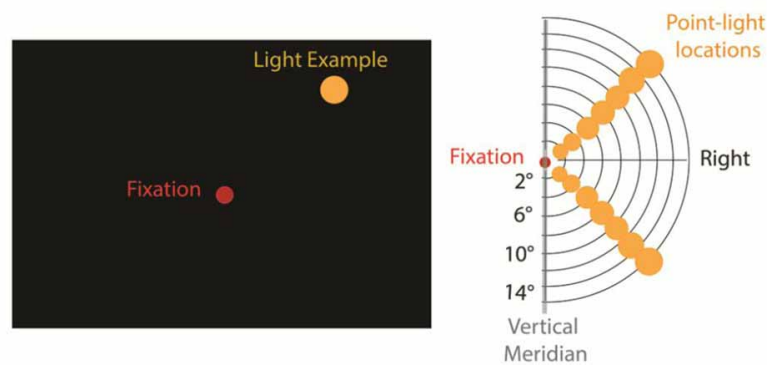

FIGURE 1 | Stimulus presentation and Apparatus. We used a standard $\mathrm{MRI}$ video projection system, back projected to a screen viewed by the participants through a monitor. A red fixation point was displayed continuously at center and single point yellow light stimuli were presented in the upper or lower right visual field at polar eccentricities ranging from 2 to $15^{\circ}$. Standard stimuli $(85 \%)$ were flashed at $8 \mathrm{~Hz}$ and target stimuli requiring a button press (15\%) were flashed at $14 \mathrm{~Hz}$.

\section{MRI DATA ACOUISITION AND ANALYSIS}

MRI data were acquired using a Siemens Allegra (3-Tesla) magnetic resonance imaging system (Siemens Medical Systems) equipped with a transmit/receive volume head coil. Head movement was restricted using a vacuum pillow and side cushioning. Blood oxygenation level dependent (BOLD) images were acquired with an echo-planar imaging sequence (32 slices, interleaved acquisition, $3 \mathrm{~mm}^{2}$ in-plane resolution, $3 \mathrm{~mm}$ thickness, no inter-slice gap, $T R=2000 \mathrm{~ms}, T E=30 \mathrm{~ms}$ ) with a $192 \mathrm{~mm}$ field of view. Axial slices were oriented in the transverse plane roughly parallel with the base of the brain. Each functional scan lasted for $5.5 \mathrm{~min}$ and consisted of 165 acquisitions. Each scanning session was composed of eight independent functional runs. For anatomical localization, high-resolution $\left(1 \mathrm{~mm}^{3}\right) \mathrm{T} 1$ weighted images of the whole brain were acquired, using a 3-D Magnetization-Prepared Rapid Acquisition Gradient echo sequence (3-D MP-RAGE, 50\% distance factor, $T R=2500 \mathrm{~ms}$, $T E=4.38 \mathrm{~ms})$. The total time a participant spent in the scanner per experimental session was typically $1-1.5$ hours.

Image processing was performed using FEAT, a part of the FMRIB Software Library (FSL) analysis suite (Smith et al., 2004). Functional image preprocessing included: motion correction using MCFLIRT, EPI non-brain removal using BET, $8 \mathrm{~mm}$ smoothing, high-pass temporal filtering (cutoff $=0.01 \mathrm{~Hz}$ ) and affine coregistration to the anatomical scan (Jenkinson et al., 2002; Smith, 2002). Each participant's anatomical volume was stripped of non-brain voxels and coregistered to the MNI 152 subject brain template using a $12^{\circ}$ of freedom affine model in FLIRT, FSL's linear coregistration tool (Jenkinson and Smith, 2001; Jenkinson et al., 2002). Brain extractions, where portions of cortex were inadvertently removed or remaining skull were identified via manual inspection and reprocessed following adjusted BET search parameters and/or manual segmentation using Space Software (http://lcni.uoregon.edu/ dow/Space_program.html). Task-related regressors were modeled at each visual-field location with each visual stimulus represented by a box-car (duration $3.125 \mathrm{~s}$ ) that was then convolved with the FSL canonical hemodynamic response function (Gamma function, delay $=6 \mathrm{~s}$, standard deviation $=3 \mathrm{~s}$ ).

\section{INDIVIDUAL HESCHL'S GYRUS REGION OF INTEREST (ROI) ANALYSIS}

To investigate whether primary auditory cortex was sensitive to increasing eccentricity and whether any variation was greater in deaf than hearing participants, we performed anatomical parcellations of Heschl's gyrus in individual brains following the methods described in our previous study (Karns et al., 2012). A caveat in both studies is that estimating primary auditory cortex boundaries based on gross anatomical landmarks may include a small portion of other auditory cortex fields (Morosan et al., 2001; Da Costa et al., 2011) however tonotopic methods are also susceptible to this caveat (Da Costa et al., 2011) and cannot be used with profoundly deaf participants. Because of considerable morphological variability across participants, anatomical segmentation is more precise than spatial coregistration and atlas-based determinations of Heschl's gyrus in group-analyses (Karns et al., 2012). Each Heschl's gyrus was parcellated by hand by raters blind to deaf or hearing status on a structural volume (T1-weighted MPRAGE) coregistered and resampled to the $2 \times 2 \times 2 \mathrm{~mm}$ FSL standard brain (MNI 152); ROIs were drawn using Space Software (http://lcni.uoregon.edu/ dow/Space_program.html). On sagittal planes, an initial boundary of Heschl's gyrus was drawn and in cases where there was a double Heschl's gyrus, both anterior and posterior gyri were included in the parcellation. These boundaries were projected onto coronal planes and adjusted if the gyrus was visible in a cross section at either sagittal or coronal orientation. The boundaries were also checked in projection on the axial planes, where voxels with low neighborhood support were excluded and voxels with high neighborhood support included. The individual Heschl's gyri were divided into Anterior and Posterior subdivisions by positioning a vertical plane oriented along the principle axis of voxel centers to allow comparison to tonotopic functional neuroimaging demonstrating human primary cortical areas A1 and R respect anatomical boundaries of anterior and posterior Heschl's gyrus, respectively. A second rater validated all parcellations and if there were any discrepancies the brain was re-entered into the set with a new subject number to be parcellated again by the original rater, who was unaware that they were re-rating a brain that they had previously parcellated. Any remaining disagreements in parcellation decisions were discussed and a final parcellation decision was made based on the protocol described above. The parameter estimates from all un-thresholded voxels within the boundary were extracted for the contrast between peripheral and perifoveal visual stimulation and scaled to percent signal change. We performed a repeated measures ANOVA (2 Hemispheres [ContralateralLeft, Ipsilateral-Right] $\times 2$ Heschl's Gyrus Subregions [Anterior, Posterior]) with Group (Deaf, Hearing) as a between subjects factor.

\section{PERIPHERAL VISUAL PROCESSING: WHOLE-BRAIN GROUP ANALYSES}

Group analyses were performed using the FSL analysis suite. Individual anatomical volumes were aligned to the Montreal Neurological Institute average template (ICBM152) using FLIRT affine coregistration. The resultant transformation was applied 
to all spatially smoothed EPI volumes to standardize functional data. Group analyses were performed using FLAME mixed effects error propagation with statistical thresholding reported for $\mathrm{Z}>2.3$ and corrected for multiple comparisons using a cluster probability thresholding of $p=0.05$ (Worsley et al., 1992; Beckmann et al., 2003; Woolrich et al., 2004). To test whether differences between deaf and hearing increased as eccentricity increased, the contrasts for each experiment were $11-15^{\circ}$ vs. $2-7^{\circ}$. Further analyses were conducted in each group alone.

\section{RESULTS}

\section{HESCHL'S GYRUS REGION OF INTEREST}

Percent signal change in anatomically-defined Heschl's gyrus was measured for the $11-15^{\circ}$ vs. $2-7^{\circ}$ contrast. Heschl's gyrus was divided into an anterior and posterior subregion (Figure 2A) to approximate primate primary-auditory areas $\mathrm{A} 1$ and $\mathrm{R}$ respectively (Da Costa et al., 2011). As shown in Figure 2B, hearing individuals had decreased signal in Heschl's gyrus for peripheral compared to perifoveal stimulation-represented as a negative signal change. In contrast, deaf individuals showed a positive signal increase for peripheral visual stimulation relative to perifoveal. Differences between deaf and hearing manifested as a Subregion $\times$ Group Interaction $\left[F_{(1,15)}=6.6, p=0.02\right]$. Follow up $t$-tests indicated that the deaf had a larger signal difference between peripheral and perifoveal locations than the hearing in Contralateral Anterior Heschl's Gyrus $\left[T_{(15)}=1.81,0.045\right.$, onetailed] and Contralateral Posterior Heschl's Gyrus $\left[T_{(15)}=1.83\right.$, $p=0.044]$ and tended to be larger in the Ipsilateral Posterior subregion $\left[T_{(15)}=1.66, p=0.059\right]$. These results indicate an increase in $H G$ signal with increasing visual eccentricity from $2-7^{\circ}$ to $11-15^{\circ}$ in the deaf but not hearing participants.

\section{GROUP ANALYSES}

Group-level analyses were performed to identify regions that showed differential recruitment to peripheral vs. perifoveal visual presentation. As shown in Figure $\mathbf{3 A}$ and detailed in Table 1, for peripheral $11-15^{\circ}$ vs. perifoveal $2-7^{\circ}$ stimuli, deaf participants showed greater activation than hearing participants in left superior-temporal auditory and multisensory cortex as well as brain regions that have also been associated with attention [left posterior parietal cortex (PPC), and anterior cingulate/SEF] $(Z>$ 2.3, $p<0.05$ corrected).

In cluster-corrected analyses performed separately within each group, the hearing participants did not show any regions with a larger response to peripheral $\left(11-15^{\circ}\right)$ vs. perifoveal $\left(2-7^{\circ}\right)$ stimuli. In contrast, deaf participants recruited higher order visual areas (contralateral MT+/V5), contralateral auditory, and multisensensory superior temporal cortex, and attention-related brain regions (left PPC, left FEF) as shown in Figure 3B and Table 2. Within each group, we did not observe significant signal increase in anterior calcarine sulcus.

\section{DISCUSSION}

The present study reveals a network of brain regions exhibiting enhanced responsiveness to peripheral visual stimuli in profoundly, genetically, and congenitally deaf adults. It is particularly noteworthy, given existing controversies (see Bavelier and Neville, 2002; Karns et al., 2012), that we found that individual anatomically-defined Heschl's gyrus regions, the site of human primary auditory cortex, showed a reliable increased response to peripheral vs. perifoveal visual stimulation in deaf participants. Greater signal in Heschl's gyrus occurred for both the anterior and posterior division (putative analog to primate A1 and R, respectively). Additionally, we found that regions

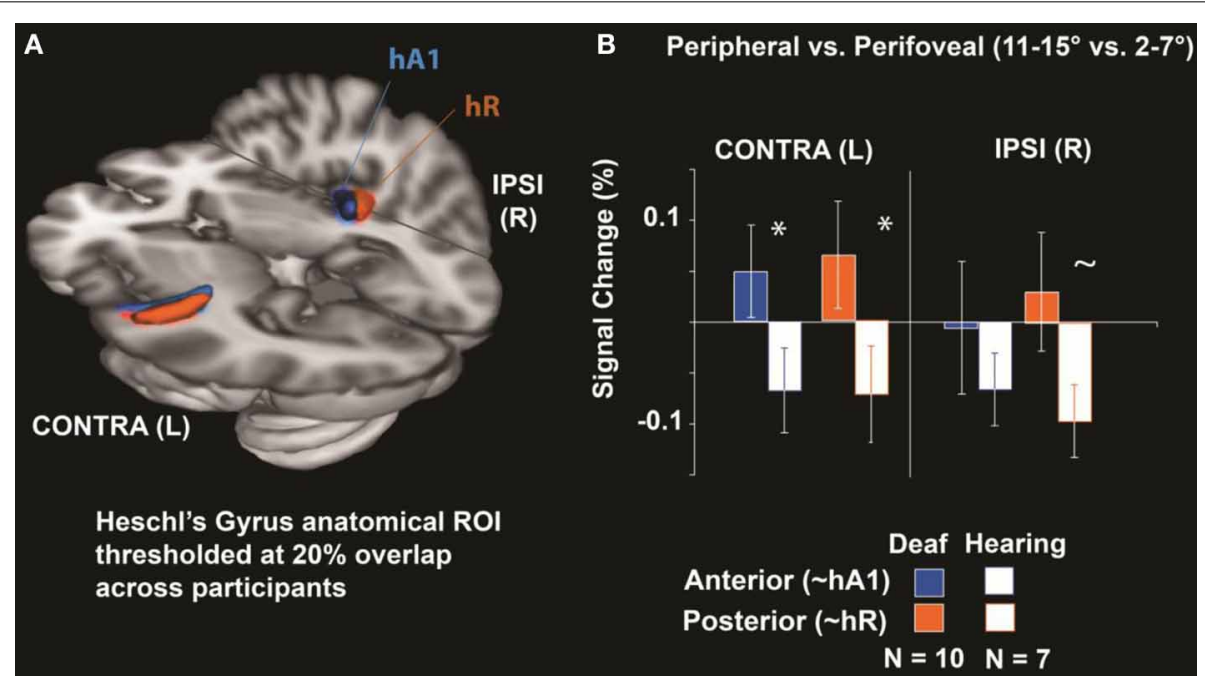

FIGURE 2 | Heschl's Gyrus Region of interest analysis. (A) Anatomical Heschl's gyrus ROls drawn on individual structural brain images divided along the first principal component axis into an anterior and posterior subregion to approximate primate primary-auditory areas $A 1$ and $R$ respectively (as defined tonotopically in hearing adults; Da Costa et al., 2011), shown here thresholded at $20 \%$ overlap across participants.
(B) Differences between peripheral $\left(11-15^{\circ}\right)$ and perifoveal locations $\left(2-7^{\circ}\right)$ were significantly larger in deaf than hearing participants for anterior and posterior Heschl's gyrus contralateral to visual stimulation $(* p<0.05)$ and tended to be larger in deaf participants posterior Heschl's gyrus ipsilateral to stimulation $(\sim p<0.10)$. Error bars represent \pm s.e.m. 


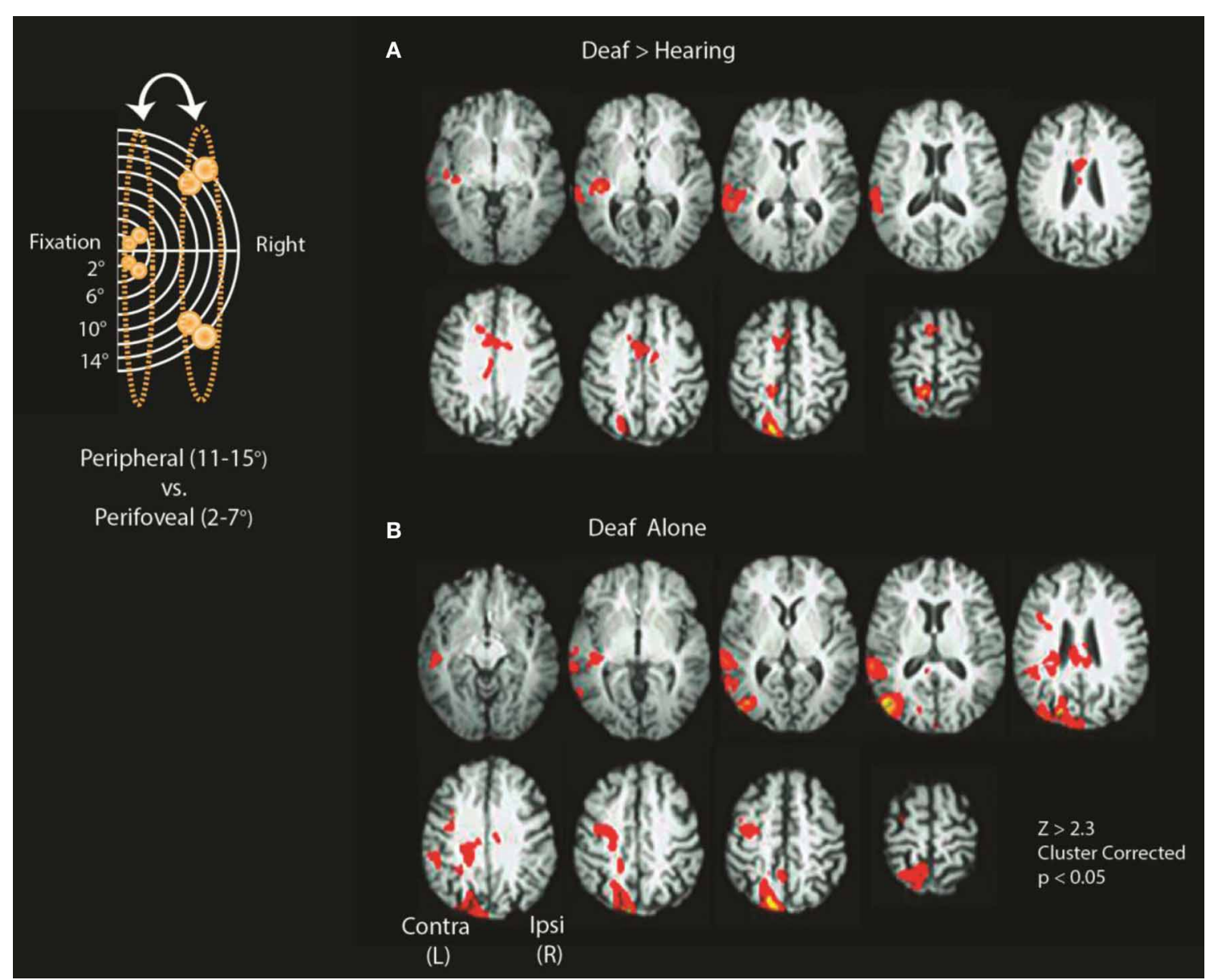

FIGURE 3 | Differences between deaf and hearing for peripheral vs. perifoveal stimulation. Inset shows a schematic location of stimuli included in the contrasts $\left(11-15^{\circ}\right.$ vs. $\left.2-7^{\circ}\right)$. (A) Deaf $>$ Hearing and (B) Deaf alone. See Tables 1, 2 for a summary of significant clusters and corresponding atlas-based descriptions.

supporting higher order visual processing (MT+/V5), as well as well as multimodal areas implicated in multisensory integration and attention (STS, PPC, anterior cingulate/SEF, and FEF) (Levanen et al., 1998; McCullough et al., 2005) which showed greater signal change for peripheral visual processing in deaf participants. Taken together, these data suggest that neuroplasticity supporting enhanced peripheral visual processing in congenital deafness involves recruitment of low level sensory cortex that has been deprived of its default sensory modality, as well as network-level recruitment of cortices involved in attention.

\section{PRIMARY AUDITORY CORTEX}

Evidence from complimentary methodology indicates that early deafness affects the anatomy of Heschl's gyrus. Structurally, auditory cortex in the deaf exhibits significantly less white matter, larger gray matter-white matter ratios, and a steeper increase in the ascending ramus of the left posterior sylvian fissure compared to hearing participants (Emmorey et al., 2003; Meyer et al., 2007; Shibata, 2007) as well as microstructural differences in fractional anisotropy, radial diffusivity, and axial diffusivity (Kim et al., 2009; Li et al., 2011; Miao et al., 2013). These findings alone might imply that Heschl's gyrus would be lessresponsive to stimulation in general due to atrophy and functional analyses such as those in the present study are critical to determine the extent to which these regions demonstrate cross-modal neuroplasticity.

Due to methodological limitations, until recently it was not clear whether Hecshl's gyrus responded to cross-modal stimulation in deafness. Studies of altered organization in deaf participants in the visual modality have generally reported crossmodal altered organization caudal to rather than overlapping Heschl's gyrus (reviewed in Bavelier et al., 2006) and studies that did report primary auditory cross-modal responses in deafness did not define Heschl's gyrus individually (Fine et al., 2005; Auer et al., 2007). It is important to define Heschl's gyrus individually due to it's high level of anatomical variability. More specifically, using a group analysis with low spatial overlap could lead to an inability to detect true responses at the individual level. Conversely, low anatomical precision due to using atlas-based localization of Heschl's gyrus could lead to misattribution of neighboring superior temporal cortex to Heschl's gyrus responses. The role of primary auditory cortex in crossmodal plasticity was recently highlighted in our 
Table 1 | Periphery (11-15) vs. central (2-7): deaf > hearing.

\begin{tabular}{|c|c|c|c|c|c|c|}
\hline \multirow[t]{2}{*}{ Hemi } & \multirow[t]{2}{*}{$Z^{*}$} & \multicolumn{3}{|c|}{ Coordinates } & \multirow[t]{2}{*}{ Harvard oxford atlas** } & \multirow[t]{2}{*}{ Juelich ${ }^{*}$} \\
\hline & & $\boldsymbol{x}$ & $y$ & $z$ & & \\
\hline $\mathrm{L}$ & 3.88 & -18 & -80 & 46 & $71 \%$ Superior LOC & $16 \% \mathrm{SPL}$ \\
\hline$L$ & 3.64 & -16 & -40 & 50 & $11 \%$ Precuneus, $10 \%$ posterior cingulate & $27 \% \mathrm{SPL}$ \\
\hline$L$ & 3.51 & -16 & -40 & 54 & $26 \%$ Postcentral G. & $28 \%$ Prim. SS, 28\% Prim. motor \\
\hline$L$ & 3.44 & -22 & -62 & 56 & $42 \%$ Superior LOC & $77 \%$ SPL 7A \\
\hline$L$ & 3.41 & -12 & -52 & 64 & $22 \%$ SPL, 19\% precuneous, $14 \%$ postcentral G. & $54 \%$ SPL 5, 38\% SPL 7A \\
\hline$L$ & 3.17 & -16 & -48 & 56 & $18 \%$ Postcentral G., 12\% SPL & $40 \%$ SPL 5, 24\% SPL 7A \\
\hline$L$ & 3.52 & -6 & 10 & 62 & $25 \%$ Superior frontal G. & $48 \%$ Premotor BA6 \\
\hline $\mathrm{R}$ & 3.1 & 2 & 4 & 36 & $92 \%$ Anterior cingulate & \\
\hline $\mathrm{L}$ & 3.07 & -6 & 4 & 22 & & $69 \%$ Callosal body \\
\hline$L$ & 3.03 & -2 & 2 & 42 & $71 \%$ Anterior cingulate & $6 \%$ Premotor BA6 \\
\hline \multirow[t]{2}{*}{$\mathrm{L}$} & 3.01 & -6 & -12 & 28 & $9 \%$ Anterior cingulate & 83\% Callosal body \\
\hline & 3.01 & 0 & 6 & 22 & $3 \%$ Anterior cingulate & $85 \%$ Callosal body \\
\hline$L$ & 3.57 & -66 & -34 & 0 & $43 \%$ pMTG, 43\% pSTG & $10 \%$ Inferior parietal lobule \\
\hline $\mathrm{L}$ & 3.44 & -64 & -20 & 8 & $41 \%$ Planum temporale, $27 \%$ pSTG & $17 \%$ Secondary SS \\
\hline $\mathrm{L}$ & 3.35 & -52 & -30 & 6 & $31 \%$ Planum temporale & $16 \%$ Prim. auditory \\
\hline$L$ & 3.32 & -58 & -38 & 6 & $58 \%$ pSTG & \\
\hline$L$ & 3.31 & -46 & -26 & -4 & $27 \%$ Heschl's G., 24\% planum temporale & 40\% Prim. auditory \\
\hline $\mathrm{L}$ & 3.26 & -48 & -26 & 2 & $22 \%$ Planum temporale, 8\% Heschl's G. & 27\% Prim. auditory \\
\hline
\end{tabular}

${ }^{*}$ Survives cluster correction, $Z>2.3, p<0.05$

** Most probable locations.

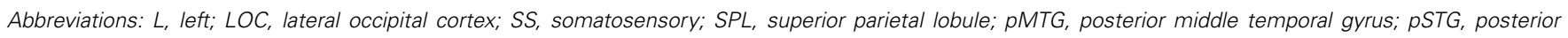
superior temporal gyrus; Prim., primary; Hemi., hemisphere; G., gyrus.

Table 2 | Periphery (11-15) vs. central (2-7): deaf alone.

\begin{tabular}{|c|c|c|c|c|c|c|}
\hline \multirow[t]{2}{*}{ Hemi } & \multirow[t]{2}{*}{$Z^{*}$} & \multicolumn{3}{|c|}{ Coordinates } & \multirow{2}{*}{$\begin{array}{l}\text { Harvard oxford } \\
\text { atlas** }\end{array}$} & \multirow[t]{2}{*}{ Juelich** } \\
\hline & & $\boldsymbol{x}$ & $y$ & $z$ & & \\
\hline L & 4.43 & -50 & -86 & 2 & $17 \%$ Inferior LOC & \\
\hline L & 4.24 & -16 & -80 & 48 & $\begin{array}{l}66 \% \text { Superior } \\
\text { LOC }\end{array}$ & $20 \%$ SPL 7 \\
\hline L & 4.24 & -50 & -74 & 8 & $1 \%$ LOC & \\
\hline L & 4.09 & -22 & -86 & 28 & $\begin{array}{l}48 \% \text { Superior } \\
\text { LOC, } 10 \% \\
\text { occipital pole }\end{array}$ & \\
\hline $\mathrm{L}$ & 4.05 & -10 & -86 & 44 & $\begin{array}{l}42 \% \text { Superior } \\
\text { LOC }\end{array}$ & $19 \% \mathrm{SPL}$ \\
\hline L & 4 & -46 & -80 & 16 & $\begin{array}{l}49 \% \text { Superior } \\
\text { LOC, } 34 \% \text { inferior } \\
\text { LOC }\end{array}$ & $\begin{array}{l}44 \% \\
\text { Inferioe } \\
\text { parietal } \\
\text { lobule (PGp) }\end{array}$ \\
\hline
\end{tabular}

${ }^{*}$ Survives cluster correction, $Z>2.3, p<0.05$.

${ }^{*}$ Most probable locations.

Abbreviations: L, left; LOC, lateral occipital cortex; SPL, superior parietal lobule; G., gyrus.

report showing somatosensory responses in anatomically-defined Heschl's gyrus of deaf participants, with the response amplitude correlated with a somatosensory double-flash illusion in the deaf (Karns et al., 2012). In animal studies, tracing also demonstrates a critical period for auditory cortex reorganization associated with the somatosensory modality (Allman et al., 2009) with potentially intact auditory connectivity in deaf ferrets (Meredith et al., 2012). In other words, in spite of atypical structure of Heschl's gyrus in deafness, it responds to cross-modal stimulation and plays a role in neuroplasticity and cortical reorganization.

The current study expands upon our previous work that demonstrates a functional link between the auditory cortex and cross-modal processing in the deaf. Although our recent report demonstrated visual responses in Heschl's gyrus (Karns et al., 2012), those responses were compared to a resting fixation baseline and thus stimulus properties and attention requirements were different across the contrast. Other reports in addition to our own also support the idea that visual responses in temporal cortices reflect increased signal relative to an implicit baseline rather than differential deactivation (Vachon et al., 2013). Given these findings, in the current study we focused our experimental design on comparing perifoveal stimulation to peripheral stimulation with target detection and visual stimulation at all stimulus locations to elucidate differences between perifoveal and peripheral stimulation. In addition, MRI background sounds are inherent to the MRI technique and cannot be matched between deaf and hearing groups, but requiring attention to the task for both peripheral and perifoveal stimulation may mitigate the potential confound of hearing people paying more attention to scanner noises during resting fixation. In spite of these methodological differences between our previous report and the present one, we replicate several observations in this non-overlapping sample of congenitally deaf adults. First, the magnitude of the unimodal 
visual response in Heschl's gyrus for deaf participants was similar across the two studies ( $\sim 0.1 \%$ signal change $)$ even though the comparisons were different (peripheral $11-15^{\circ}$ vs. perifoveal $2-7^{\circ}$ target detection in the current experiment and far peripheral $45^{\circ}$ visual stimulation vs. central resting fixation in our previous experiment). It is important to note that somatosensory responses in our previous study were considerably larger $(\sim 0.3 \%$ signal change) than visual stimulation. Both studies showed a more robust response in the Heschl's gyrus contralateral to visual stimulation, but a limitation is that stimulation in both studies always occurred on the right and future studies need to address whether this generalizes to left visual field stimulation. Nonetheless, the similarities across the results from two studies that used different stimulation methods and different participants is reassuring.

If visual stimulation recruits primary auditory cortex in deaf participants, particularly for the peripheral visual field, it is important to consider how this altered organization arises. While traditionally assigned to low-level auditory processing, more recently primary auditory cortex has also been linked to multisensory functionality and neuroplasticity in humans (see Levanen et al., 1998; Fine et al., 2005; Schroeder and Foxe, 2005; Musacchia and Shroeder, 2009). Stabilization of cross-modal connections is one potential mechanism; if cross-modal connections are typical in the auditory cortex of hearing individuals these connections could increase when acoustic input is reduced.

Although in the present study we have focused on primary auditory cortex, an important question is the degree to which low-level visual or primary visual cortex is differentially recruited for peripheral visual processing in congenitally deaf adults. Provocative EEG data may demonstrate differences between deaf and hearing individuals in early visual cortical ERP components $80 \mathrm{~ms}$ after stimulus onset (Bottari et al., 2011) but the degree to which preparatory potentials influence early-latency visual responses remain to be elucidated. While our group-level analyses have not shown differences in primary visual cortex, the calcarine sulcus, like Heschl's gyrus, has high variability across individuals and approaches with more anatomical specificity may show differences in deafness. Future studies will address this deficiency.

\section{MULTISENSORY AND HIGHER ORDER BRAIN REGIONS}

In addition to enhancements in lower-level auditory areas, deaf participants in the present study displayed a crossmodal neuroplasticity in a number of regions including MT+/V5, STS, PPC, and anterior cingulate/SEF and FEF. Previous studies have linked these regions to visuospatial attention (Nobre et al., 1997; Buchel et al., 1998; Beauchamp et al., 2001; Serences et al., 2005; Saygin and Sereno, 2008) as well as multisensory integration (Hagen et al., 2002; Laurienti et al., 2003; Beauchamp et al., 2004; Blake et al., 2004; Woldoff et al., 2004; Mulette-Gillman et al., 2005; Mukai et al., 2007). Previous research also showed that MT+/V5 exhibits increased activation in the deaf in response to attended stimuli particularly in the periphery (Bavelier et al., 2000, 2001) and STS and PPC exhibits enhanced recruitment in deaf participants with attention (Bavelier et al., 2000, 2001). Our data shows that this network is differentially recruited for peripheral stimulation in deaf participants despite use of simple visual stimuli; this suggests that the common function of these brain regions in visuospatial attention and multisensory integration may be a determining factor in the enhancements they display in the deaf.

Previous research in animal models has shown that there can be high specificity in terms of which brain regions support enhanced visual performance in early deafness. In early deaf cats, reversible deactivation of posterior auditory cortex selectively impaired their enhanced visual localization performance, while deactivation of the dorsal auditory cortex impacted their visual motion detection, suggesting that it is possible to localize individual visual functions in specific parts of reorganized auditory cortex (Lomber et al., 2010). What is not clear from this study is whether other higher-order brain regions, such as attention networks, also support the enhanced visual performance in deaf animals. Our whole-brain analysis suggests that in humans, other brain regions also support processing of peripheral visual stimuli in deafness. What remains to be elucidated is how these regions interact to support peripheral processing. For example, one TMSfMRI study in hearing humans reported that FEF imparts unique functional connectivity to primary visual areas representing the periphery (Ruff et al., 2008). This raises the question of whether FEF is an upstream cause of differential anterior visual cortex activation in the deaf or a downstream target of heightened anterior visual cortex activation. Future research using methods such as TMS or resting-state functional connectivity could address the interactions of these regions.

\section{Population considerations}

A possible confounding variable in visual neuroplasticity studies comparing congenitally deaf to hearing individuals is early acquisition of sign language. Studies that include native hearing signers can separate the neural changes associated with early signlanguage acquisition from those linked to auditory deprivation. However, most of the effects that have been attributed to sign language acquisition concern the pattern of hemispheric laterality. Hearing native signers, for example, exhibit motion detection asymmetries favoring the right visual field and temporal-parietal event-related brain potential asymmetries favoring the left hemisphere similar to congenitally deaf participants and opposite to those displayed by hearing non-signing individuals (Neville and Lawson, 1987c; Bosworth and Dobkins, 2002b). Both hearing and deaf native signers viewing moving stimuli also exhibited unique left lateralization of activation in the motion-related brain areas, MT+/V5-MST (Bavelier et al., 2001). Experiments using more complex language-oriented stimuli found unique righthemispheric activation bias in native deaf and hearing signers that is dependent on the age of acquisition of sign language (Bavelier et al., 1998; Neville et al., 1998; Newman et al., 2001). Data from the current study likely reflects the effect of auditory deprivation rather than sign language acquisition since previous research has demonstrated that hearing native signers do not display the superior motion detection, visual attention, or lower visual field advantages seen in the deaf, nor the enhanced recruitment of A1, MT+/V5, STS, or parietal cortex that deaf individuals do (Neville and Lawson, 1987c; Bavelier et al., 2001; Bavelier and Neville, 2002). 


\section{CONCLUSION}

Our data indicate that the peripheral visual field is an important sensory domain of crossmodal neuroplasticity in the deaf that involves multiple brain regions. These regions range from those classically considered unisensory such as the primary auditory cortex, to higher-level associative areas. Many of the interconnected regions share a functional role in multisensory integration and visuospatial attention. Further research along these lines will contribute fundamental information about the mechanisms, specificity, and constraints of human neuroplasticity. While such information contributes to our basic understanding of neuroplasticity, it can also be harnessed to guide the development and refinement of educational and rehabilitative programs for typically and non-typically developing individuals.

\section{ACKNOWLEDGMENTS}

This publication was made possible by Grant Number R01 DC000128 from National Institutes of Health, National Institute on Deafness, and other Communication Disorders to Helen J. Neville. Magnetic resonance imaging was performed at the Robert and Beverly Lewis Center for Neuroimaging at the University of Oregon.

\section{REFERENCES}

Allman, B., Keniston, L., and Meredith, M. (2009). Adult deafness induces somatosensory conversion of ferret auditory cortex. Proc. Natl. Acad. Sci. U.S.A. 106, 5925-5930. doi: 10.1073/pnas.0809483106

Auer, E. T. Jr., Bernstein, L., Sungkarat, W., and Singh, M. (2007). Vibrotactile activation of the auditory cortices in deaf versus hearing adults. Neuroreport 18, 645-648. doi: 10.1097/WNR.0b013e3280d943b9

Bavelier, D., Brozinsky, C., Tomann, A., Mitchell, T., Neville, H., and Liu, G. (2001). Impact of early deafness and early exposure to sign language on the cerebral organization for motion processing. J. Neurosci. 21, 8931-8942.

Bavelier, D., Corina, D., Jezzard, P., Clark, V., Karni, A., Lalwani, A., et al. (1998). Hemispheric specialization for English and ASL: Left invariance - right variability. Neuroreport 9, 1537-1542. doi: 10.1097/00001756-199805110-00054

Bavelier, D., Dye, M., and Hauser, P. (2006). Do deaf individuals see better? Trends Cogn. Sci. 10, 512-518. doi: 10.1016/j.tics.2006.09.006

Bavelier, D., and Neville, H. J. (2002). Cross-modal plasticity: Where and how? Nat. Rev. Neurosci. 3, 443-452.

Bavelier, D., Tomann, A., Hutton, C., Mitchell, T., Corina, D., Liu, G., et al. (2000). Visual attention to the periphery is enhanced in congenitally deaf individuals. J. Neurosci. 20, 1-6.

Beauchamp, M., Argall, B., Bodurka, J., Duyn, J., and Martin, A. (2004). Unraveling multisensory integration: Patchy organization within human STS multisensory cortex. Nat. Neurosci. 7, 1190-1192. doi: 10.1038/nn1333

Beauchamp, M., Petit, L., Ellmore, T., Ingeholm, J., and Haxby, J. V. (2001). A parametric fMRI study of overt and covert shifts of visuospatial attention. Neuroimage 14, 310-321. doi: 10.1006/nimg.2001.0788

Beckmann, C., Jenkinson, M., and Smith, S. (2003). General multi-level linear modeling for group analysis in fMRI. Neuroimage 20, 1052-1063. doi: 10.1016/S1053-8119(03)00435-X

Blake, R., Sobel, K., and James, T. (2004). Neural synergy between kinetic vision and touch. Psychol. Sci. 15, 397-402. doi: 10.1111/j.0956-7976.2004. 00691.x

Bosworth, R. G., and Dobkins, K. R. (2002a). The effects of spatial attention on motion processing in deaf signers, hearing signers, and hearing nonsigners. Brain Cogn. 49, 152-169. doi: 10.1006/brcg.2001.1497

Bosworth, R. G., and Dobkins, K. R. (2002b). Visual field asymmetries for motion processing in deaf and hearing signers. Brain Cogn. 49, 170-181. doi: 10.1006/brcg.2001.1498

Bottari, D., Caclin, A., Giard, M., and Pavani, F. (2011). Changes in early cortical visual processing predict enhanced reactivity in deaf individuals. PLOS ONE 6:e25607. doi: 10.1371/journal.pone.0025607
Bridge, H., Clare, S., Jenkinson, M., Jezzard, P., Parker, A., and Matthews, P. (2005). Independent anatomical and functional measures of the v1/v2 boundary in human visual cortex. J. Vis. 5, 93-102. doi: 10.1167/5.2.1

Buchel, C., Josephs, O., Rees, G., Turner, R., Frith, C., and Friston, K. (1998). The functional anatomy of attention to visual motion: a functional MRI study. Brain 121, 1281-1294. doi: 10.1093/brain/121.7.1281

Da Costa, S., Van Der Zwaag, W., Marques, J., Frackowiak, R. S. J., Clarke, S., and Saenz, M. (2011). Human primary auditory cortex follows the shape of Heschl's gyrus. J. Neurosci. 31, 14067-14075. doi: 10.1523/JNEUROSCI.2000-11. 2011

Di Russo, F., Martinez, A., Sereno, M., Pitzalis, S., and Hillyard, S. (2001). Cortical sources of the early components of the visual evoked potential. Hum. Brain Mapp. 15, 95-111. doi: 10.1002/hbm.10010

Emmorey, K., Allen, J., Bruss, J., Schenker, N., and Damasio, H. (2003). A morphometric analysis of auditory brain regions in congenitally deaf adults. Proc. Natl. Acad. Sci. U.S.A. 100, 10049-10054. doi: 10.1073/pnas. 1730169100

Fine, I., Finney, E. M., Boynton, G. M., and Dobkins, K. R. (2005). Comparing the effects of auditory deprivation and sign language within the auditory and visual cortex. J. Cogn. Neurosci. 17, 1621-1637. doi: 10.1162/08989290577 4597173

Finney, E. M., Fine, I., and Dobkins, K. R. (2001). Visual stimuli activate auditory cortex in the deaf. Nat. Neurosci. 4, 1171-1173. doi: 10.1038/nn763

Hackett, T. (2011). Information flow in the auditory cortical network. Hear. Res. 27, 133-146. doi: 10.1016/j.heares.2010.01.011

Hagen, M., Franzen, O., McGlone, F., Essick, G., Dancer, C., and Pardo, J. (2002). Tactile motion activates the human middle temporal / V5 (MT+/V5) complex. Eur. J. Neurosci. 16, 957-964. doi: 10.1046/j.1460-9568.2002.02139.x

Jenkinson, M., Bannister, P., Brady, M., and Smith, S. (2002). Improved optimization for the robust and accurate linear registration and motion correction of brain images. Neuroimage 17, 825-841. doi: 10.1006/nimg.2002.1132

Jenkinson, M., and Smith, S. (2001). A global optimisation method for robust affine registration of brain images. Med. Image Anal. 5, 143-156. doi: 10.1016/S13618415(01)00036-6

Karns, C., Dow, M., and Neville, H. (2012). Altered cross-modal processing in the primary auditory cortex of congenitally deaf adults: a visual-somatosensory fMRI study with a double-flash illusion. J. Neurosci. 32, 9626-9638. doi: 10.1523/JNEUROSCI.6488-11.2012

Kim, D.-J., Park, S.-Y., Kim, J., Lee, D., and Park, H.-J. (2009). Alterations of white matter diffusion anisotropy in early deafness. Neuroreport 20, 1032-1036. doi: 10.1097/WNR.0b013e32832e0cdd

Kral, A., Shröder, J.-H., Klinke, R., and Engel, A. (2003). Absence of cross-modal reorganization in the primary auditory cortex of congenitally deaf cats. Exp. Brain Res. 153, 605-613. doi: 10.1007/s00221-003-1609-Z

Laurienti, P., Wallace, M., Maldjian, J., Susi, C., Stein, B., and Burdette, J. (2003). Cross-modal sensory processing in the anterior cingulate and medial fronta cortices. Hum. Brain Mapp. 19, 212-223. doi: 10.1002/hbm.10112

Levanen, S., Jousmaki, V., and Hari, R. (1998). Vibration-induced auditory cortex activation in a cogenitally deaf adult. Curr. Biol. 8, 869-872. doi: 10.1016/S09609822(07)00348-X

Li, Y., Ding, G., Booth, J., Huang, R., Lv, Y., He, Y., et al. (2011). Sensitive period for white-matter connectivity of superior temporal cortex in deaf people. Hum. Brain Mapp. 33, 349-359. doi: 10.1002/hbm.21215

Loke, W. H., and Song, S. (1991). Central and peripheral visual processing in hearing and nonhearing individuals. Bull. Psychon. Soc. 29, 437-440. doi: 10.3758/BF03333964

Lomber, S., Meredith, M., and Kral, A. (2010). Cross-modal plasticity in specific auditory cortices underlies visual compensations in the deaf. Nat. Neurosci. 13, 1421-1427. doi: 10.1038/nn.2653

Lu, H., Basso, G., Serences, J., Yantis, S., Golay, X., and Van Zijl, P. (2005). Retinotopic mapping in the human visual cortex using vascular space occupancy-dependent functional magnetic resonance imaging. Neuroreport 16, 1635-1640. doi: 10.1097/01.wnr.0000181580.18636.ee

McCullough, S., Emmorey, K., and Sereno, M. (2005). Neural organization for recognition of grammatical and emotional facial expressions in deaf ASL signers and hearing nonsigners. Brain Res. 22, 193-203. doi: 10.1016/j.cogbrainres.2004.08.012

Meredith, A., Keniston L.P., and Allman, B. (2012). Multisensory dysfunction accompanies crossmodal plasticity following adult hearing impairment. Neuroscience 214, 136-148. doi: 10.1016/j.neuroscience.2012.04.001 
Meredith, M., Allman, B., Keniston, L., and Clemo, H. (2009). Auditory influences on non-auditory cortices. Hear. Res. 258, 64-71. doi: 10.1016/j.heares.2009.03.005

Meredith, M., and Lomber, S. (2011). Somatosensory and visual crossmodal plasticity in the anterior auditory field of early-deaf cats. Hear. Res. 280, 38-47. doi: 10.1016/j.heares.2011.02.004

Meyer, M., Toepel, U., Keller, J., Nussbaumer, D., Zysset, S., and Friederici, A. (2007). Neuroplasticity of sign language: implications from structural and functional brain imaging. Restor. Neurol. Neurosci. 25, 335-351.

Miao, W., Li, J., Tang, M., Xian, J., Li, W., Liu, Z., et al. (2013). Altered white matter integrity in adolescents with prelingual deafness: a high-resolution tractbased spatial statistics imaging study. Am. J. Neuroradiol. 34, 1264-1270. doi: 10.3174/ajnr.A3370

Morosan, P., Rademacher, J., Schleicher, A., Amunts, K., Schormann, T., and Zilles, K. (2001). Human primary auditory cortex: cytoarchitectonic subdivisions and mapping into a spatial reference system. Neuroimage 13, 684-701. doi: 10.1006/nimg.2000.0715

Mukai, I., Kim, D., Fukunaga, M., Japee, S., Marrett, S., and Ungerleider, L. G. (2007). Activations in visual and attention-related areas predict and correlate with the degree of perceptual learning. J. Neurosci. 27, 11401-11411. doi: 10.1523/JNEUROSCI.3002-07.2007

Mulette-Gillman, O., Cohen, Y., and Groh, J. (2005). Eye-centered, head-centered, and complex coding of visual and auditory targets in the intraparietal sulcus. J. Neurophysiol. 94, 2331-2352. doi: 10.1152/jn.00021.2005

Musacchia, G., and Shroeder, C. (2009). Neuronal mechanisms, response dynamics, and perceptual functions of multisensory interactions in auditory cortex. Hear. Res. 258, 72-79. doi: 10.1016/j.heares.2009.06.018

Neville, H., Bavelier, D., Corina, D., Rauschecker, J., Karni, A., Lalwani, A., et al. (1998). Cerebral organization for language in deaf and hearing subjects: biological constraints and effects of experience. Proc. Natl. Acad. Sci. U.S.A. 95, 922-929. doi: 10.1073/pnas.95.3.922

Neville, H., and Lawson, D. (1987a). Attention to central and peripheral visual space in a movement detection task: an event-related potential and behavioral study. I. Normal hearing adults. Brain Res. 405, 253-267. doi: 10.1016/00068993(87)90295-2

Neville, H., and Lawson, D. (1987b). Attention to central and peripheral visual space in a movement detection task: an event-related potential and behaviora study. II. Congenitally deaf adults. Brain Res. 405, 268-283. doi: 10.1016/00068993(87)90296-4

Neville, H., and Lawson, D. (1987c). Attention to central and peripheral visual space in a movement detection task: an event-related potential and behavioral study. III. Separate effects of auditory deprivation and acquisition of a visual language. Brain Res. 405, 284-294. doi: 10.1016/0006-8993(87)90297-6

Neville, H., Schmidt, A., and Kutas, M. (1983). Altered visual-evoked potentials in congenitally deaf adults. Brain Res. 266, 127-132. doi: 10.1016/00068993(83)91314-8

Newman, A., Bavelier, D., Corina, D., Jezzard, P., and Neville, H. (2001). A critical period for right hemisphere recruitment in American Sign Language processing. Nature 5, 76-80.

Nobre, A., Sebestyen, G., Gitelman, D., Mesulam, M., Frackowiak, R. S. J., and Frith, C. (1997). Functional localization of the system for visuospatial attention using postron emission tomography. Brain 120, 515-533. doi 10.1093/brain/120.3.515

Ruff, C., Bestmann, S., Blankenburg, F., Bjoerto, O., Josephs, O., Weiskopf, N., et al. (2008). Distinct causal influences of parietal versus frontal areas on human visual cortex: Evidence from concurrent TMS-fMRI. Cereb. Cortex 18, 817-827. doi: $10.1093 /$ cercor/bhm 128
Saygin, A., and Sereno, M. (2008). Retinotopy and attention in human occiptal, temporal, parietal, and frontal cortex. Cereb. Cortex 18, 2158-2168. doi: $10.1093 /$ cercor/bhm 242

Schroeder, C., and Foxe, J. (2005). Multisensory contributions to lowlevel, 'unisensory' processing. Curr. Opin. Neurobiol. 15, 454-458. doi: 10.1016/j.conb.2005.06.008

Serences, J., Shomstein, S., Leber, A., Golay, X., Egeth, H., and Yantis, S. (2005) Coordination of voluntary and stimulus-driven attentional control in human cortex. Psychol. Sci. 16, 114-122. doi: 10.1111/j.0956-7976.2005.00791.x

Shibata, D. (2007). Differences in brain structure in deaf persons on MR imaging studied with voxel-based morphometry. Am. J. Neuroradiol. 28, 243-249.

Smith, S. (2002). Fast robust automated brain extraction. Hum. Brain Mapp. 17, 143-155. doi: 10.1002/hbm.10062

Smith, S., Jenkinson, M., Woolrich, M., Beckmann, C., Behrens, T., JohansenBerg, H., et al. (2004). Advances in functional and structural MR image analysis and implementation as FSL. Neuroimage 23, S208-S219. doi: 10.1016/j.neuroimage.2004.07.051

Stevens, C., and Neville, H. (2006). Neuroplasticity as a double-edged sword: Deaf enhancements and dyslexic deficits in motion processing. J. Cogn. Neurosci. 18, 701-714. doi: 10.1162/jocn.2006.18.5.701

Tootell, R. B., Hadjikhani, K., Hall, E., Marrett, S., Vanduffel, W., Vaughan, J., et al. (1998a). The retinotopy of visual spatial attention. Neuron 21, 1409-1422. doi: 10.1016/S0896-6273(00)80659-5

Tootell, R. B., Hadjikhani, K., Vanduffel, W., Liu, A., Mendola, J., Sereno, M., et al. (1998b). Functional analysis of primary visual cortex (V1) in humans. Proc Natl. Acad. Sci. U.S.A. 95, 811-817. doi: 10.1073/pnas.95.3.811

Vachon, P., Voss, P., Lassonde, M., Leroux, J.-M., Mensour, B., Beaudoin, G., et al. (2013). Reorganization of the auditory, visual and multimodal areas in early deaf individuals. Neuroscience 245, 50-60. doi: 10.1016/j.neuroscience.2013.04.004

Woldoff, M., Hazlett, C., Fichtenholtz, H., Weissman, D., Dale, A., and Song, A. (2004). Functional parcellation of attentional control regions of the brain. J. Cogn. Neurosci. 16, 149-165. doi: 10.1162/089892904322755638

Woolrich, M., Behrens, T., Beckmann, C., Jenkinson, M., and Smith, S. (2004) Multi-level linear modelling for fMRI group analysis using Bayesian inference. Neuroimage 21, 1732-1747. doi: 10.1016/j.neuroimage.2003. 12.023

Worsley, K., Evans, A., Marrett, S., and Neelin, P. (1992). A three-dimensional statistical analysis for CBF activation studies in human brain. J. Cereb. Blood Flow Metab. 12, 900-918. doi: 10.1038/jcbfm.1992.127

Conflict of Interest Statement: The authors declare that the research was conducted in the absence of any commercial or financial relationships that could be construed as a potential conflict of interest.

Received: 13 January 2014; accepted: 10 March 2014; published online: 26 March 2014. Citation: Scott GD, Karns CM, Dow MW, Stevens C and Neville HJ (2014) Enhanced peripheral visual processing in congenitally deaf humans is supported by multiple brain regions, including primary auditory cortex. Front. Hum. Neurosci. 8:177. doi: 10.3389/ fnhum.2014.00177

This article was submitted to the journal Frontiers in Human Neuroscience.

Copyright (c) 2014 Scott, Karns, Dow, Stevens and Neville. This is an open-access article distributed under the terms of the Creative Commons Attribution License (CC BY). The use, distribution or reproduction in other forums is permitted, provided the original author(s) or licensor are credited and that the original publication in this journal is cited, in accordance with accepted academic practice. No use, distribution or reproduction is permitted which does not comply with these terms. 